\title{
2D-SAXS in situ Measurements as a Tool to Study Elusive Mesoporous Phases: The Case of $p 6 \mathrm{~mm} \mathrm{TiO}$
}

Estefania Gonzalez Solveyra, María Cecilia Fuertes, Galo J. A. A. Soler-Illia, and Paula C. Angelomé J. Phys. Chem. C, Just Accepted Manuscript • DOI: 10.1021/acs.jpcc.6b12112 • Publication Date (Web): 18 Jan 2017 Downloaded from http://pubs.acs.org on January 27, 2017

\section{Just Accepted}

"Just Accepted" manuscripts have been peer-reviewed and accepted for publication. They are posted online prior to technical editing, formatting for publication and author proofing. The American Chemical Society provides "Just Accepted" as a free service to the research community to expedite the dissemination of scientific material as soon as possible after acceptance. "Just Accepted" manuscripts appear in full in PDF format accompanied by an HTML abstract. "Just Accepted" manuscripts have been fully peer reviewed, but should not be considered the official version of record. They are accessible to all readers and citable by the Digital Object Identifier (DOI®). "Just Accepted" is an optional service offered to authors. Therefore, the "Just Accepted" Web site may not include all articles that will be published in the journal. After a manuscript is technically edited and formatted, it will be removed from the "Just Accepted" Web site and published as an ASAP article. Note that technical editing may introduce minor changes to the manuscript text and/or graphics which could affect content, and all legal disclaimers and ethical guidelines that apply to the journal pertain. ACS cannot be held responsible for errors or consequences arising from the use of information contained in these "Just Accepted" manuscripts. 


\title{
2D-SAXS In Situ Measurements as a Tool to Study Elusive Mesoporous Phases: the Case of p6mm $\mathrm{TiO}_{2}$
}

\author{
Estefania Gonzalez Solveyra ${ }^{1 \square}$, M. Cecilia Fuertes ${ }^{2,3}$, Galo J. A. A. Soler-Illia ${ }^{1,4}$, Paula C. \\ Angelomé ${ }^{*}$
}

\begin{abstract}
(1) Departamento de Química Inorgánica, Analítica y Química Física, Facultad de Ciencias Exactas y Naturales, Universidad de Buenos Aires, Pabellón II, Ciudad Universitaria, C1428EHA, Buenos Aires, Argentina.
\end{abstract}

(2) Gerencia Química, Centro Atómico Constituyentes, Comisión Nacional de Energía Atómica, CONICET, Av. Gral Paz 1499, (B1650KNA) San Martín, Buenos Aires, Argentina.

(3) Instituto Sabato, UNSAM, CNEA. Av. Gral. Paz 1499, (B1650KNA) San Martín, Buenos Aires, Argentina.

(4) Instituto de Nanosistemas, UNSAM, CONICET Av. 25 de Mayo y Francia, (1650) San Martín, Buenos Aires, Argentina.

$(\square)$ Current Address: Department of Biomedical Engineering, Department of Chemistry and Chemistry of Life Processes Institute, Northwestern University, Evanston, IL, United States

* Corresponding author email: angelome@cnea.gov.ar 


\begin{abstract}
$\underline{\text { Abstract }}$
Mesoporous titania thin films (MTTF) with $\mathrm{p} 6 \mathrm{~mm}$ pore array are currently attracting a great interest because of their 2D-hexagonal arrange of close-packed cylindrical pores, but are difficult to synthesize. This work seeks to understand such apparent elusiveness of the $p 6 \mathrm{~mm}$ phase for MTTF. To that end, a wide variety of MTTF were prepared using different precursors, templates, aging and stabilization conditions, according to procedures reported in the literature and several variations thereof. Initially, the obtained MTTFs were cross-characterized by ex situ TEM, 2D-SAXS and GISAXS, with no clear evidence of a well organized $p 6 \mathrm{~mm}$ pore structure in the final system. In view of the difficulty of obtaining the desired pore array, the mesophase evolution was monitored during the synthesis, aging, stabilization and calcination stages, resorting to in situ 2D-SAXS experiments. The performed experiments indicate that the key parameters that affect the ordering in these systems are the humidity of conservation but, mainly, the thermal treatment. Among the different strategies rehearsed, the desired mesophase was finally obtained by a combination of high humidity aging, exposure to $\mathrm{NH}_{3}$ vapors and a thermal treatment at low temperature, followed by ethanol extraction of the template. However, even resorting to such mild treatments, the obtained MTTF presented ordered $p 6 \mathrm{~mm}$ structure combined with disordered regions, as determined by electron microscopy and porosimetry measurements.

Thus, the obtained results stress that considering the mesophase obtained only at initial or intermediate synthesis stages as the one that survives in the template-free system is not a safe assumption. Moreover, complementary experimental techniques should be used to determine mesoporous structures in the final systems. Finally, this work shows the strength of in situ 2DSAXS measurements as a technique to design new synthetic procedures, in order to obtain specific properties of the final material.
\end{abstract}




\section{Introduction}

Mesoporous $\mathrm{TiO}_{2}$ thin films (MTTF) have attracted a great deal of attention in the last years, due to the combination of interesting properties from the material and from its structure. In fact, $\mathrm{TiO}_{2}$ is a wide band gap semiconductor and as such presents optical and electronic properties that allow its use as a photocatalyst and as a part of photovoltaic devices. ${ }^{1}$ Adding porosity to $\mathrm{TiO}_{2}$ increases its surface area, allowing the use of smaller amounts of material, and enhances its reactivity by boosting surface interactions. The controlled size and order of the mesopores (in the 2-10 $\mathrm{nm}$ range) allows the control over the species that can diffuse through its structure. Also, obtaining the material as a thin film permits easy integration with well established techniques to prepare more complex multiscale devices. Hence, the potential applications of such engineered MTTF include optical, electronic, and electrochemical devices, batteries, sensors and catalyst supports. ${ }^{2-6}$

The first examples of MTTF were reported in $2001^{7,8}$ and since then several methodologies have been presented in the literature to obtain this material. ${ }^{6,9,10}$ Among all the reported approaches, the most widely used is the Evaporation Induced Self Assembly (EISA). ${ }^{11} \mathrm{~A}$ typical EISA process starts with the preparation of an acidic alcohol rich solution containing stabilized $\mathrm{Ti}$ species and the chosen template. From this solution, mesostructured $\mathrm{TiO}_{2}$ hybrid thin films are obtained, generally by spin or dip coating. Subsequently, the films are subjected to an aging process that allows the formation of a robust and highly organized mesostructure, with organic and inorganic domains well interdispersed. Finally, a thermal treatment or an extraction method is used to eliminate the template in order to obtain the final MTTF ${ }^{6}$. This MTTF can also be obtained with crystalline anatase walls, if the proper thermal treatment and substrate are used. ${ }^{10,12}$

The obtained pore array of the MTTF depends on the identity of the template, its concentration and the processing conditions; ${ }^{13}$ the most commonly obtained arrays are depicted in Scheme 1. 


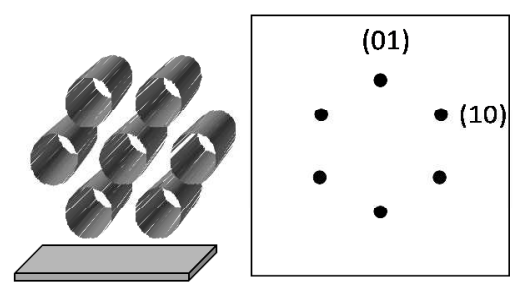

2D hex

p6mm

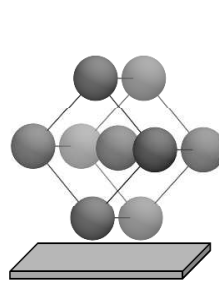

Cubic Im3m

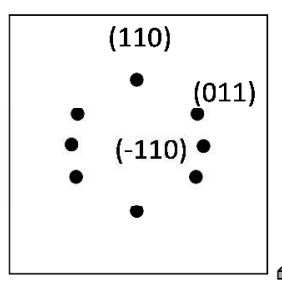

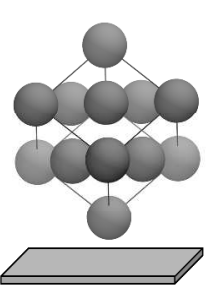

Cubic

Fm3m

Scheme 1: Commonly obtained porous arrays for MTTF, its orientation and their 2D-SAXS theoretical patterns.

Molecular transport through mesoporous structures depends on the obtained pore array architecture, as demonstrated by Hillhouse's group for mesoporous $\mathrm{SiO}_{2}$ thin films. ${ }^{14}$ They showed that the ordering and orientation of the pores can be a dominant factor to determine the transport and the degree to which an electrode under the film is accessible. In particular, well-ordered $p 6 \mathrm{~mm}$ structures with mesopores oriented parallel to the surface can be highly blocking ${ }^{14}$. Also, the photocatalytic activity of $\mathrm{TiO}_{2} \mathrm{MTTF}$ was demonstrated to depend on tridimensional structure of the oxide. ${ }^{15}$ In particular, it was shown that the pore architecture affects the adsorption affinity toward guest molecules as well as the accessibility, adsorption, and diffusion behavior of guest molecules within the pore network. ${ }^{15}$ Rankin and coworkers also determined that small changes in pore orientation have a substantial impact on the evolution of mesostructural order and crystallinity degree during thermal treatment. ${ }^{16,17}$

Among all the possible ordered arrays of mesopores that can be obtained (see Scheme 1), ${ }^{9,} 10$ mesoporous titania thin films with $p 6 \mathrm{~mm}$ pore array are currently attracting a great interest since they provide a 2D-hexagonal arrange of close-packed packed cylindrical pores. This interest is driven both by fundamental science and practical applications: when guest molecules are introduced into the oriented mesochannels, macroscopically anisotropic properties can be developed. Thus, $p 6 \mathrm{~mm}$ thin films are strongly demanded for the construction of molecular-scale devices and as platforms to study diffusion and confinement inside mesopores. ${ }^{3}$ Also, interconnected pores (as obtained in other MTTF structures, such as cubic Im3m) may not be desirable in certain applications, since the presence of bottle necks can considerably slow down liquids infiltration rate and affect the diffusion behavior of guest molecules. ${ }^{18}$ Mesoporous thin films with independent cylindrical pores also provide "ideal" systems to perform adsorption/desorption studies, given their characteristic isotherms and 
hysteresis loops. ${ }^{19}$ The sorption properties of a system give information about its textural properties: specific area, pore volume, pore size distribution, network connectivity. ${ }^{20}$ Hence, the knowledge derived from such ideal systems can be later applied to rationalize the sorption behavior in more complex mesoporous structures, improving their characterization. ${ }^{21}$ In addition, fully oriented mesoporous titania-based thin films presenting a 2D-hexagonal anisotropic mesostructure can exhibit remarkable birefringence as well as a high refractive index porous matrix; this structural anisotropy can find applications in optical devices such as highly stable phase plates. ${ }^{22,23}$

The $p 6 \mathrm{~mm}$ phase can be easily obtained for the case of mesoporous $\mathrm{SiO}_{2}$ thin films. This phase has been presented in several papers, accompanied with an adequately characterization by complementary techniques. ${ }^{8,14,24,25}$ However, $\mathrm{TiO}_{2}$ thin films with $p 6 \mathrm{~mm}$ pore arrangement have been less studied and, in general, the characterization of the final material (after elimination of the template) was not presented or was done based only on electron microscopy techniques. ${ }^{13,26-30}$ It should be mentioned here that the information provided by such techniques, although highly valuable, is local, and as such should only be used as further confirmation of results obtained by diffraction techniques, such as Small Angle X-Ray Scattering (2D-SAXS) or Grazing incidence SAXS (GISAXS). ${ }^{10}$ Also, it has been demonstrated that a pure $p 6 \mathrm{~mm}$ structure is difficult to obtain in titania systems ${ }^{16,31}$ and that the alignment of the pores critically determines the stability of the mesophase towards thermal treatments. ${ }^{17}$

In this work, we set to understand the apparent elusiveness of the $p 6 \mathrm{~mm}$ phase for mesoporous $\mathrm{TiO}_{2}$. To that end, in situ 2D-SAXS technique was used to follow the changes undergone by the thin film mesostructure during different synthesis, aging, stabilization and calcination procedures. This technique provides the full diffraction patterns of the MTTF, from which the space group, the lattice parameters and the pore array orientation can be obtained. Additionally, as the data are collected sampling at millimetre scale area, the results are representative of the whole sample. ${ }^{10}$ In situ 2D-SAXS experiments have been previously used to follow the structural evolution during mesoporous thin film deposition and aging steps, in particular to follow the formation of mesoporous $\mathrm{SiO}_{2}{ }^{8,32-35}$ and $\mathrm{TiO}_{2}{ }^{13,31,33}$. In this line, some recent works by Rankin and coworkers have dealt with the thermal evolution of MTTF prepared onto different substrates ${ }^{16,17}$; and also some previous works have been dedicated to study the effect of $\mathrm{TiO}_{2}$ crystallization onto the film's mesostructure. ${ }^{36}$ Similarly, the in situ experiments performed in this work explore the initial stages, but also expand further to study the effect of 
different post-synthesis processes until the final mesoporous (template free) material is obtained.

A wide variety of MTTF using different precursors, templates, aging conditions and stabilization treatments (thermal and vapor treatments) were prepared, in the quest of a well ordered 2D-hexagonal template free MTTF. Procedures reported in the literature were used as a starting point, and variations of those procedures were also performed. The results are organized in three sections. In the first section, the mesoporous structure of the obtained MTTF was characterized by ex situ electronic microscopies, 2D-SAXS and GISAXS. In view of the difficulty of obtaining $p 6 \mathrm{~mm}$ structures, in situ 2D-SAXS experiments were design in order to monitor the mesophase evolution during the film synthesis and processing stages. The results of such experiments are presented and discussed in the second section. Finally, given the importance of using complementary experimental techniques to determine mesoporous structures, further characterization of the $p 6 \mathrm{~mm}$ samples obtained from the in situ experiments is presented in the last section. The results presented are critically discussed and analysed, and the main findings of our work are summarized in the final conclusions.

\section{Experimental section}

\section{Materials}

Titanium tetraethoxide $\left(\mathrm{Ti}(\mathrm{OEt})_{4}\right)$, titanium tetrachloride $\left(\mathrm{TiCl}_{4}\right)$, Pluronic $\mathrm{F} 127$ $\left(\mathrm{HO}\left(\mathrm{CH}_{2} \mathrm{CH}_{2} \mathrm{O}\right)_{106}\left(\mathrm{CH}_{2} \mathrm{CH}\left(\mathrm{CH}_{3}\right) \mathrm{O}\right)_{70}\left(\mathrm{CH}_{2} \mathrm{CH}_{2} \mathrm{O}\right)_{106} \mathrm{OH}\right)$ and Pluronic 123 $\left(\mathrm{HO}\left(\mathrm{CH}_{2} \mathrm{CH}_{2} \mathrm{O}\right)_{20}\left(\mathrm{CH}_{2} \mathrm{CH}\left(\mathrm{CH}_{3}\right) \mathrm{O}\right)_{70}\left(\mathrm{CH}_{2} \mathrm{CH}_{2} \mathrm{O}\right)_{20} \mathrm{OH}\right)$ were supplied by Sigma-Aldrich, and used as received. $\mathrm{HCl}$ and Brij $56\left(\mathrm{C}_{16} \mathrm{H}_{33}\left(\mathrm{CH}_{2} \mathrm{CH}_{2} \mathrm{O}\right)_{10} \mathrm{OH}\right)$ were purchased from Merck. Pure grade ethanol and 1-butanol were used as solvents. E-pure water was used to prepare the sols and to clean the material.

\section{Mesoporous thin films synthesis}

MTTF were prepared according to protocols reported in the literature as a starting point, and several variations thereof. The parameters varied included the source of Ti(IV), the template, the composition of the sol, and the conditions of the stabilization, consolidation and calcination steps (temperature, duration of the treatment, relative humidity). All systems studied, alongside with the references where they were first reported, are collected in Table 1 and Table S1 in the SI. The general protocols for the preparation of the sols and the films are as follows. 
Sol preparation: All sols were prepared using a $\mathrm{Ti}(\mathrm{IV})$ compound $\left(\mathrm{TiCl}_{4}\right.$ or $\left.\mathrm{Ti}(\mathrm{OEt})_{4}\right)$ as the inorganic source, a template (F127, Brij 56 or P123), water and a solvent (ethanol or 1-butanol). When $\mathrm{Ti}(\mathrm{OEt})_{4}$ was used as the precursor, $\mathrm{HCl}$ was also added to the initial mixture, to control the hydrolysis of the precursor. In general, the Ti(IV) source was initially mixed with the solvent (and $\mathrm{HCl}$ if needed), then the template was added and finally water was incorporated dropwise under stirring. In Table 1 (and Table S1 in the SI) we summarize the great variety of molar relations tested. The main parameters varied were: $s=$ [template]/[Ti], $h=\left[\mathrm{H}_{2} \mathrm{O}\right] /[\mathrm{Ti}], p=$ $[\mathrm{HCl}] /[\mathrm{Ti}]$ and $s v=[$ solvent $] /[\mathrm{Ti}]$. The sols and films were named according to their Ti(IV) source ( $\mathrm{TE}$ for $\mathrm{Ti}(\mathrm{OEt})_{4}$ and $\mathrm{TC}$ for $\left.\mathrm{TiCl}_{4}\right)$, their template $(\mathrm{F}=\mathrm{F} 127, \mathrm{P}=\mathrm{P} 123, \mathrm{~B}=$ Brij 56), their surfactantto-metal ratio $(s)$, and the solvent used $(\mathrm{E}=\mathrm{EtOH}$ and $\mathrm{B}=1-\mathrm{BuOH})$. For example, TC-F-005-E denotes the film synthesized with $s=0.005$ using $\mathrm{TiCl}_{4}$, Pluronic $\mathrm{F} 127$ and EtOH as inorganic source, template, and solvent respectively.

Film Synthesis: Glass substrates (coverslips or microscopy slides) were washed using deionized water and rinsed with acetone or ethanol. Thin films were prepared on the unmodified substrates by dip coating at a constant withdrawal rate between 1 to $4 \mathrm{~mm} \mathrm{~s}^{-1}$, under controlled relative humidity $(\mathrm{RH})$. Alternatively, the films were produced by spin-coating, depositing $125 \mu \mathrm{L}$ of the sol at a spinning rate of $6000 \mathrm{rpm}$. After deposition, the films were stabilized for different times in chambers with controlled $\mathrm{RH}$ obtained using $\mathrm{Ca}\left(\mathrm{NO}_{3}\right)_{2}$ saturated solution for $50 \% \mathrm{RH}$, $\mathrm{KNO}_{3}$ saturated solution for $80 \% \mathrm{RH}$ and $\mathrm{MgCl}_{2}$ saturated solution for $30 \% \mathrm{RH}$. The temperature of the stabilization stage was also controlled at approximately $25^{\circ} \mathrm{C}$ (room temperature) or $-5{ }^{\circ} \mathrm{C}$ (refrigerator temperature). After stabilization, the films were consolidated resorting to thermal treatments at $60^{\circ} \mathrm{C}$ and $130^{\circ} \mathrm{C}$ or to ammonia or water vapor treatments. In the final calcination step, the films were thermally treated with one or more consecutive steps from room temperature up to $200{ }^{\circ} \mathrm{C}-400{ }^{\circ} \mathrm{C}$, in furnaces under a still air atmosphere. All the detailed tested conditions are described in Table $\mathbf{1}$ and Table $\mathbf{S 1}$ in the SI.

\section{Characterization}

2D Small-Angle X-ray Scattering (2D-SAXS). 2D-SAXS measurements were performed at the SAXS2 line of the Laboratório Nacional de Luz Síncrotron (LNLS, Campinas, SP, Brazil). A $\lambda=1.55$ $\AA$ was selected and the data collection was performed with a CCD detector. The beamline humidity and temperature were fixed at $50 \% \mathrm{RH}$ and $25{ }^{\circ} \mathrm{C}$, respectively. For the in situ measurements, the sample-detector distance was fixed at $733 \mathrm{~mm}$. Samples were prepared by 
spin coating in the vicinity of the beamline, and were mounted immediately after synthesis (less than two minutes) at $4^{\circ}$ incidence angle. The 2D-SAXS patterns were collected every $150 \mathrm{~s}$ up to 30 to 60 minutes, depending on the sample, using a collection time of $30 \mathrm{~s}$. For the ex situ measurements, the sample-detector distance was in the range $500-1400 \mathrm{~mm}$, and $4^{\circ}$ and $90^{\circ}$ incidence angles were used. For better understanding of the results, the most commonly obtained porous arrays for MTTF are depicted in Scheme 1, alongside their 2D-SAXS theoretical patterns.

Grazing incidence-small-angle X-ray scattering (GISAXS). GISAXS measurements were performed at the XRD2 beamline of LNLS using a six circles Huber diffractometer $(\lambda=1.5498 \AA$ ) and a Pilatus detector. The incident angle was fixed at $0.22^{\circ} \theta$ and sample-detector distance was 507 $\mathrm{mm}$.

Transmission Electron Microscopy (TEM). TEM images were collected using a Philips EM 301 transmission microscope (CMA, Facultad de Ciencias Exactas y Naturales, UBA) operated at 60 $\mathrm{kV}$. Samples were obtained by scratching the films from the substrate with a razor blade and dispersed in ethanol. A drop of that dispersion was deposited on a carbon-coated copper grid and dried before analysis.

Environmental Ellipsometric Porosimetry (EEP). Water adsorption-desorption curves (at $25{ }^{\circ} \mathrm{C}$ ) were measured by EEP (SOPRA GES5A). Film thickness and refractive index values were obtained from the ellipsometric parameters $\psi$ and $\Delta$ under nitrogen flux containing variable water vapor pressure, $\mathrm{P} ; \mathrm{P} / \mathrm{P}_{\mathrm{O}}$ was varied from 0 to $1\left(\mathrm{P}_{\mathrm{O}}\right.$ being the saturation water vapor pressure at $\left.25^{\circ} \mathrm{C}\right)$. Water volume adsorbed at each $\mathrm{P} / \mathrm{P}_{\mathrm{O}}$ value was determined by modeling the obtained refractive index according to a three-component (water-air-oxide) Bruggeman effective medium approximation (BEMA). Adsorption-desorption isotherms were obtained by plotting the water volume adsorbed by the porous film at each $\mathrm{P} / \mathrm{P}_{\mathrm{O}}$. The pore size distributions were determined using models based on the Kelvin equation on these isotherms, taking into account the water contact angle in the film. ${ }^{37}$ The contact angle of water on the films was determined by depositing a sessile droplet directly on top of the sample and captured the static contact angle using a Ramé-Hart 290-F4. Image analysis was performed with Ramé-Hart DROP image software.

\section{Results and discussion}

Ex situ experiments 
In the literature, three usual strategies are used to obtain 2D-hexagonal mesoporous $\mathrm{TiO}_{2}$, that can be summarized as follows: a) use of templates with smaller hydrophilic domains compared with the ones used for obtaining cubic structures ${ }^{13}$; b) use of a higher molar relation between template and Ti source $(s=[\text { template }] /[\mathrm{Ti}])^{13,38}$; and $\mathrm{c}$ ) use of a low temperature and high relative humidity during thin film deposition and stabilization. ${ }^{28,39}$ All three strategies were tested in this work, using as a base previously reported procedures and also testing a wide range of variations. Mainly Pluronic P123 and Brij 56 were used as templates, to reduce hydrophilicity when compared with Pluronic F127 and Brij 58, respectively; although Pluronic F127 was also tested. For those systems, the employed amount of template was higher than in the case of cubic structures: a typical $s$ of 0.005 is used for F127-templated cubic structures whereas for hexagonal phases, $s$ ranges between 0.009 and 0.01 were explored. Also different solvents (1butanol, ethanol), different amounts of water $\left(h=\left[\mathrm{H}_{2} \mathrm{O}\right] /[\mathrm{Ti}]\right.$ in the 3-10 range) and several post treatment conditions were tested. Finally, the $\mathrm{Ti}(\mathrm{IV})$ source was varied resorting to $\mathrm{TiCl}_{4}$ or $\mathrm{Ti}(\mathrm{OEt})_{4}$. All the examined conditions are summarized in Table 1. 
Table 1: Details of the sols composition of the $\mathrm{TiO}_{2}$ mesoporous thin films prepared in this work and the obtained structure (based on 2D-SAXS, GISAXS and TEM measurements). For the extended version of this table, see Table $\mathrm{S} 1$ (SI).

$s=[$ Template $] /[\mathrm{Ti}], h=\left[\mathrm{H}_{2} \mathrm{O}\right] /[\mathrm{Ti}], p=[\mathrm{HCl}] /[\mathrm{Ti}], s v=[$ solvent $] /[\mathrm{Ti}]$

$*$ = variations on a given reference

\begin{tabular}{|c|c|c|c|c|c|c|c|c|}
\hline \multirow{2}{*}{ Sample } & \multicolumn{6}{|c|}{ Sol composition } & \multirow{2}{*}{ Pore array } & \multirow{2}{*}{ Reference } \\
\hline & Template & Solvent & $s$ & $h$ & $p$ & sv & & \\
\hline TC-F-005-E & F127 & EtOH & 0.005 & 10 & - & 40 & $\operatorname{Im} 3 m$ cubic & 13 \\
\hline TC-F-009-E & F127 & $\mathrm{EtOH}$ & 0.009 & 10 & - & 40 & $\begin{array}{c}\text { Im3m cubic } \\
\quad \text { or } \\
\text { Local order }\end{array}$ & 13 \\
\hline TC-F-01-E & F127 & EtOH & 0.01 & 3 & - & 40 & Local order & 13 \\
\hline TC-F-01-E & F127 & $\mathrm{EtOH}$ & 0.01 & 5 & - & 40 & Local order & 13 \\
\hline TC-F-01-E & F127 & $\mathrm{EtOH}$ & 0.01 & 10 & - & 40 & $\begin{array}{c}\text { Im3m cubic } \\
\text { or } \\
\text { Local order }\end{array}$ & 13 \\
\hline TC-F-01-B & F127 & 1-BuOH & 0.01 & 5 & - & 40 & $\begin{array}{c}\text { Im3m cubic } \\
\text { or } \\
\text { Local order }\end{array}$ & $13,27^{*}$ \\
\hline TC-F-01-B & F127 & 1-BuOH & 0.01 & 10 & - & 40 & Local order & $13,27^{*}$ \\
\hline TC-B-05-E & Brij 56 & $\mathrm{EtOH}$ & 0.05 & 10 & - & 40 & Local order & $40,41^{*}$ \\
\hline TC-B-1-E & Brij 56 & $\mathrm{EtOH}$ & 0.1 & 10 & - & 40 & Local order & $40,41^{*}$ \\
\hline TC-B-2-E & Brij 56 & $\mathrm{EtOH}$ & 0.2 & 10 & - & 40 & Local order & 13 \\
\hline TC-B-2-E & Brij 56 & $\mathrm{EtOH}$ & 0.2 & 10 & - & 40 & Disordered & 13 \\
\hline TC-P-017-E & P123 & $\mathrm{EtOH}$ & 0.017 & 2 & - & 40 & Local order & 29,30 \\
\hline TC-P-017-E & P123 & $\mathrm{EtOH}$ & 0.017 & 3 & - & 40 & Local order & 29,30 \\
\hline TC-P-05-E & P123 & $\mathrm{EtOH}$ & 0.05 & 10 & - & 40 & Undefined & 13 \\
\hline TC-P-07-E & P123 & EtOH & 0.07 & 10 & - & 40 & Disordered & 13 \\
\hline TC-P-09-E & P123 & $\mathrm{EtOH}$ & 0.09 & 10 & - & 40 & Disordered & 13 \\
\hline TC-P-1-E & P123 & $\mathrm{EtOH}$ & 0.1 & 10 & - & 40 & $\begin{array}{l}\text { Local order } \\
\text { or } \\
\text { disordered }\end{array}$ & $13 *$ \\
\hline TC-P-025-B & P123 & 1-BuOH & 0.025 & 3 & - & 40 & Disordered & $13,27^{*}$ \\
\hline TC-P-025-B & P123 & 1-BuOH & 0.025 & 6 & - & 40 & Disordered & $13,27^{*}$ \\
\hline TC-P-025-B & P123 & 1-BuOH & 0.025 & 10 & - & 40 & Disordered & $13,27^{*}$ \\
\hline TE-P-0095-E & P123 & EtOH & 0.0095 & 6 & 1.75 & 14.5 & Local order & 26 \\
\hline TE-P-014-E & P123 & EtOH & 0.014 & 6.86 & 2 & 17 & Fm $3 m$ cubic & 28 \\
\hline TE-P-022-E & P123 & EtOH & 0.022 & 6 & 1.75 & 14.5 & Local order & 26 \\
\hline TE-P-013-B & P123 & 1-BuOH & 0.013 & 6 & 2 & 9 & Fm $3 m$ cubic & 27 \\
\hline
\end{tabular}

For all the systems discussed in this section, the samples were characterized after the final calcination treatment with the consequent template removal from the pores, using both electron microscopy and GISAXS or 2D-SAXS as characterization techniques. In Figure 1, TEM 


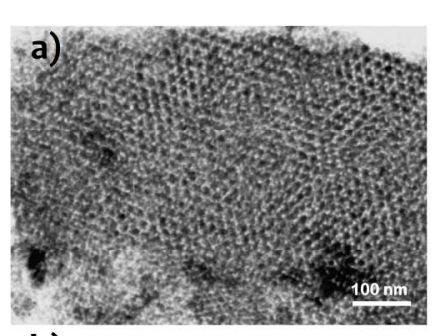

b)

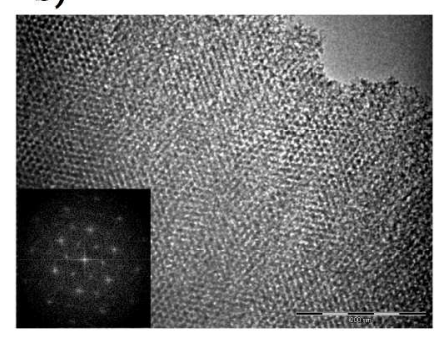

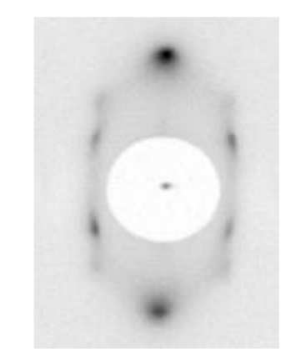

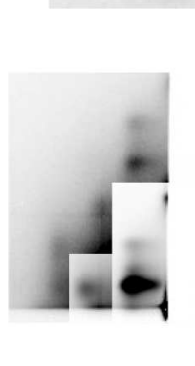

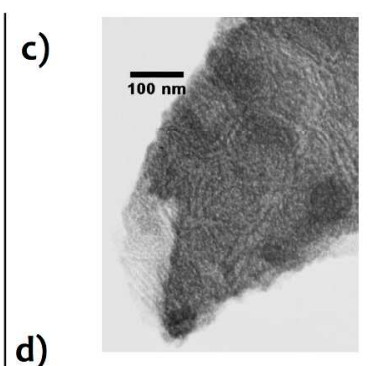

d)
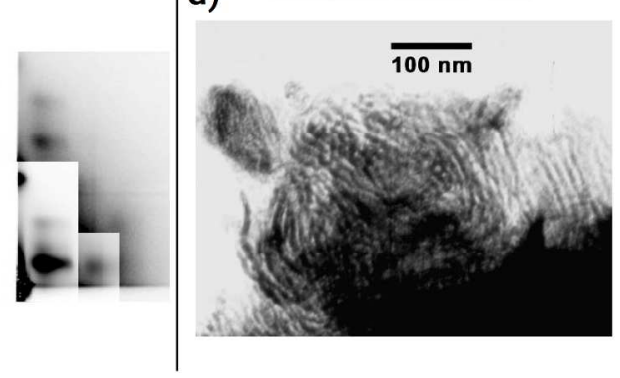

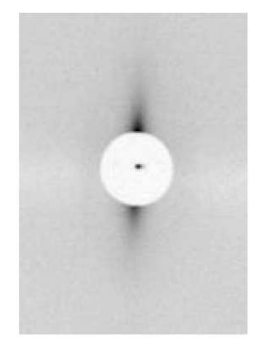

Figure 1: Ex situ characterization of $\mathrm{TiO}_{2}$ mesoporous thin films prepared using different reported protocols employing $\mathrm{P} 123$ as a directing agent to obtain the mesostructure $p 6 \mathrm{~mm}$. (a) TEM image (left) and 2D-SAXS diagram (right) of sample TE-P-013-B. (b) TEM image (left) and GISAXS diagram (right) of sample TE-P-014-E. Inset in TEM image correspond to its Fourier transform. (c) TEM image (left) and 2D-SAXS pattern (right) of sample TC-P-025-B. (d) TEM image (left) and 2D-SAXS pattern (right) of sample TE-P-022-E.

As a general trend (see Table 1), it was not possible to obtain the 2D-hexagonal phase in any of the tested conditions. Instead, $\mathrm{Fm} 3 \mathrm{~m}$ face centered cubic (see, for example, Figure $\mathbf{1} \mathbf{a}-\mathbf{b}$ ), locally ordered (see, for example, Figure 1d) or disordered phases (Figure 1c) were obtained. These results can be attributed to several factors. One of the possibilities is the incorrect attribution of the pore array order in the literature, due to the lack of crossed characterization. ${ }^{10}$ In this regard, it is also important to note that in several publications the reported 2D-SAXS (or GISAXS) patterns correspond to samples that are mesostructured but not mesoporous. That is, samples that were stabilized at low temperatures and still have the template inside the pores. All the systems discussed so far correspond to mesoporous materials, i.e., samples submitted to calcination treatments towards eliminating the surfactant molecules inside the pores (see Tables 1 and S1). This difference could imply that the $p 6 \mathrm{~mm}$ hexagonal phase is lost during 
thermal treatments at higher temperature. Finally, critical factors in the evolution of the film structure, such as the number of processing steps, the temperature, $\mathrm{RH}$ and time at each of them, are sometimes missing from the protocols reported in the literature. This could also account for the different obtained mesoporous structures. Finally, recent reports show that chemical modification of the slides could improve the stability of the $p 6 \mathrm{~mm}$ mesostructure, ${ }^{16,31}$, ${ }^{39}$ but all films studied in this work were deposited on unmodified glass surfaces.

Thus, the main conclusion of our ex situ experiments is that the $p 6 \mathrm{~mm}$ hexagonal phase is difficult to obtain and easy to lose during the processing of MTTF. Structural evolution upon film synthesis, stabilization, consolidation and template-removal depends strongly on the processing conditions in a complex manner. ${ }^{10}$ The results presented so far highlight that considering the structure observed at initial or intermediate stages, as the one that survives in the final system is not a safe assumption. As the processing parameters were identified to be key factors in obtaining the $p 6 \mathrm{~mm}$ phase, 2D-SAXS in situ experiments were planned, in order to study the formation and evolution of eventually obtained $p 6 \mathrm{~mm}$ phase as a function of the synthesis and post-synthesis conditions.

\section{In situ 2D-SAXS experiments}

For the in situ 2D-SAXS measurements, the most promising sols according to the literature and the results from the ex situ experiments were chosen to be studied. For every sample, the 2D-SAXS pattern was followed continuously after 1-2 minutes of film deposition by spin coating (performed next to the beamline) for 30 to 60 minutes. Afterwards, the films were subjected to different post-treatment conditions and measurements were performed before and after each step.

The first system tested was $\mathrm{TiCl}_{4}-\mathrm{P} 123$, with the following molar relations: $\mathrm{TiCl}_{4}$ 1: $\mathrm{P} 123$ $0.1: \mathrm{H}_{2} \mathrm{O} 10: \mathrm{EtOH}$ 40. In Figure 2, selected 2D-SAXS patterns are presented (the complete set of conditions studied can be found in Figure S2, SI). For this case, locally ordered structure is formed just after the thin film deposition. After 30 minutes of drying at room temperature and $\mathrm{RH}\left(25^{\circ} \mathrm{C}\right.$ and $50 \%$, respectively), the typical pattern of $\mathrm{p} 6 \mathrm{~mm}$ ordering was clearly observed. The diffraction spots are superimposed with a halo, which indicates the presence of some areas with locally ordered structure. After $4.5 \mathrm{~h}$ of drying in a humidity controlled chamber, the $p 6 \mathrm{~mm}$ pattern is more defined (less intensity in the halo is observed) and it is stable up to $14 \mathrm{~h}$ in the same conditions. After this stabilization at $50 \% \mathrm{RH}$, a wide variety of thermal treatments were 
tested (see Figure 2 and Figure S2). In all cases, when the stabilization temperature was increased from room temperature, the order of the structure disappeared. A stabilization of the oxide structure using $\mathrm{NH}_{3}$ vapors was also tried, ${ }^{38}$ but even in this case, it was not possible to obtain a $p 6 \mathrm{~mm}$ ordered and oriented mesostructured oxide. It is possible that the observed pattern does not correspond to the ordered oxide but to a phase segregation of pure polymer template. That could also explain the lack of uniaxial contraction during drying of the structure, ${ }^{13}$ that is usually observed for mesoporous thin films (see below). These results also indicate that the $2 \mathrm{D}$-hexagonal structure, if formed, is not stable enough to resist the thermal treatment usually used to obtain mesoporous $\mathrm{TiO}_{2}$. Probably, the stress caused by the consolidation of the oxide walls lead to a disruption of the polymer organization.

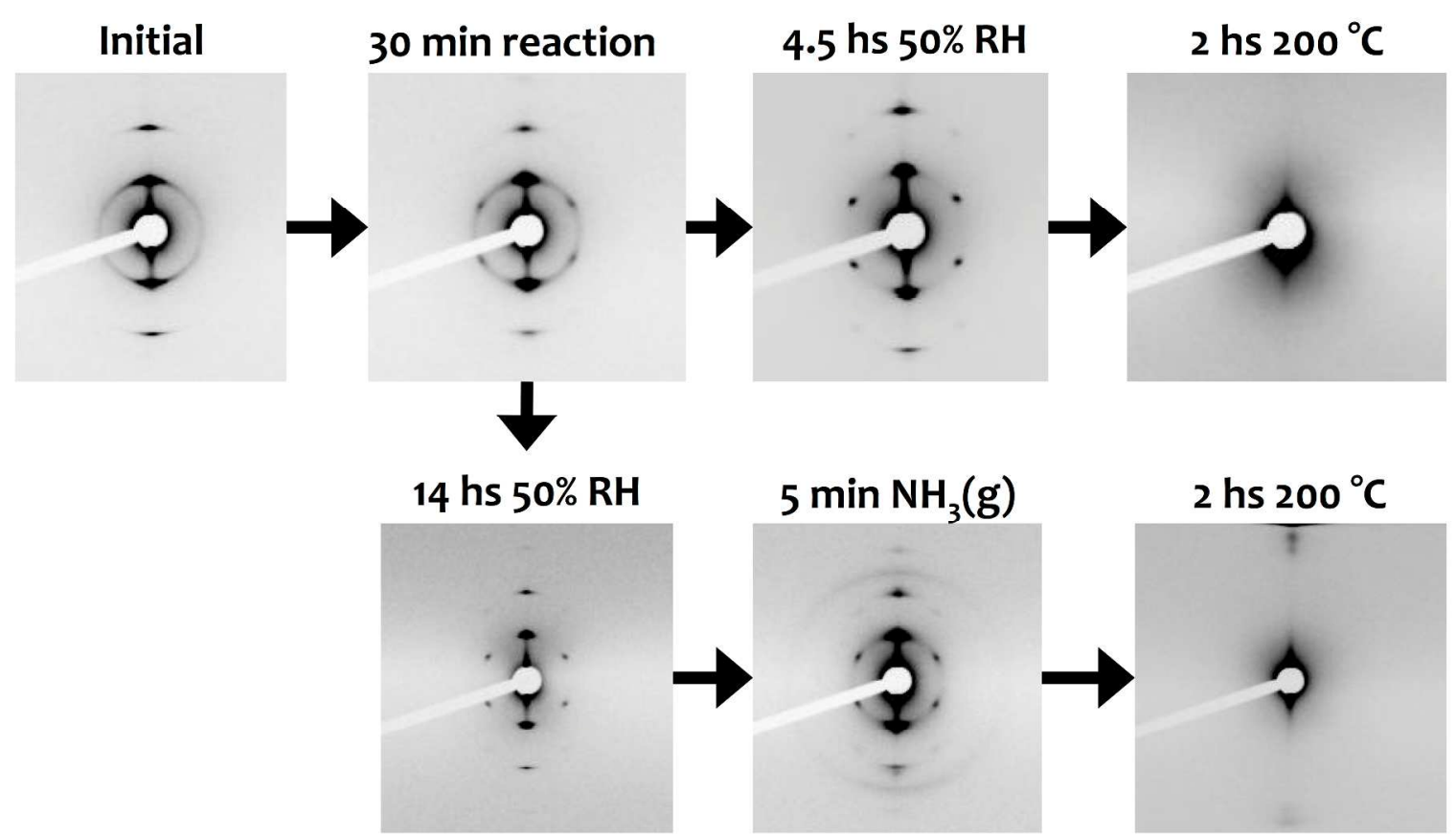

Figure 2: In situ 2D-SAXS characterization of mesoporous thin films prepared with $\mathrm{TiCl}_{4}$ and $\mathrm{P} 123$ under different conditions, as indicated in the diagram. The complete set of conditions studied can be found in Figure S2 (SI).

The second system tested was $\mathrm{TiCl}_{4}-\mathrm{Brij} 56$, with the following molar relations: $\mathrm{TiCl}_{4} 1$ : Brij56 $0.2: \mathrm{H}_{2} \mathrm{O} 10$ : EtOH 40. For this system, previous in situ studies have shown the appearance of the $p 6 \mathrm{~mm}$ phase just after the thin film deposition. ${ }^{13}$ In Figure 3, selected 2DSAXS patterns are presented and the complete set of conditions studied can be found in Figure S3. In this case, a locally ordered structure accompanied by an $\operatorname{Im} 3 m$ cubic structure is formed just after the thin film deposition, and this structure is kept after 1 hour of drying at room 
temperature and $\mathrm{RH}\left(25{ }^{\circ} \mathrm{C}\right.$ and $50 \%$, respectively). After this time, different samples were introduced in chambers with controlled humidity at $30 \%, 50 \%$ and $80 \%$. Only in the case of $80 \%$ $\mathrm{RH}$ a clear $p 6 \mathrm{~mm}$ pattern was developed after $6 \mathrm{~h}$ of aging. This result indicates that a high humidity seems to be a key parameter to obtain the 2D-hex structure, in line with recent results in the literature. ${ }^{28,39}$ Although previous work indicated that low relative humidity favored mesophases with less curvature (i.e., $p 6 \mathrm{~mm}$ over $(\mathrm{m} 3 \mathrm{~m}$ ), the presence of a higher amount of water vapor might kinetically assist to rearrange the initial cubic structure into the final hexagonal phase, since the film is still soft enough to allow it. ${ }^{13,31}$ However, the high RH is not the only factor that defines the final mesostructure, and after a direct treatment at $200{ }^{\circ} \mathrm{C}$ (Figure 3) or after a sequence treatment with $60^{\circ} \mathrm{C}$ and $130{ }^{\circ} \mathrm{C}$ steps (Figure S3, SI) the structure collapsed and no diffraction spots were observed. In order to improve the thermal stability of the mesostructured oxide, the material was submitted to a short contact with $\mathrm{NH}_{3}$ vapors. ${ }^{38}$ To obtain a well-ordered mesostructured film, the inorganic matrix should consolidate around the mesophase of the surfactant without significantly disrupting it. This balance between inorganic condensation and mesophase structuration depends critically on the precursor, template agent and processing parameters. For titania films prepared trough EISA, it has been proposed that the condensation is triggered by the evaporation of $\mathrm{HCl}^{35}$ Treatment of the mesostructured films with $\mathrm{NH}_{3}$ vapors helps to consolidate the oxide, since ammonia reacts with $\mathrm{HCl}$ present in the sol, lowering the acidity and accelerating the condensation process, without the need of thermal treatment to favor $\mathrm{HCl}$ evaporation. In this way, the inorganic network is consolidated at low temperatures while preventing the disruption of the surfactant mesophase. As a consequence, the $p 6 \mathrm{~mm}$ structure remained after both the thermal treatment up to $200^{\circ} \mathrm{C}$ and the template extraction with ethanol, as can be seen in the lower panel of Figure $\mathbf{3}$. It is also important to note that a uniaxial contraction is observed after the thermal treatment, indicating that the observed pattern corresponds to the mesoporous oxide. 


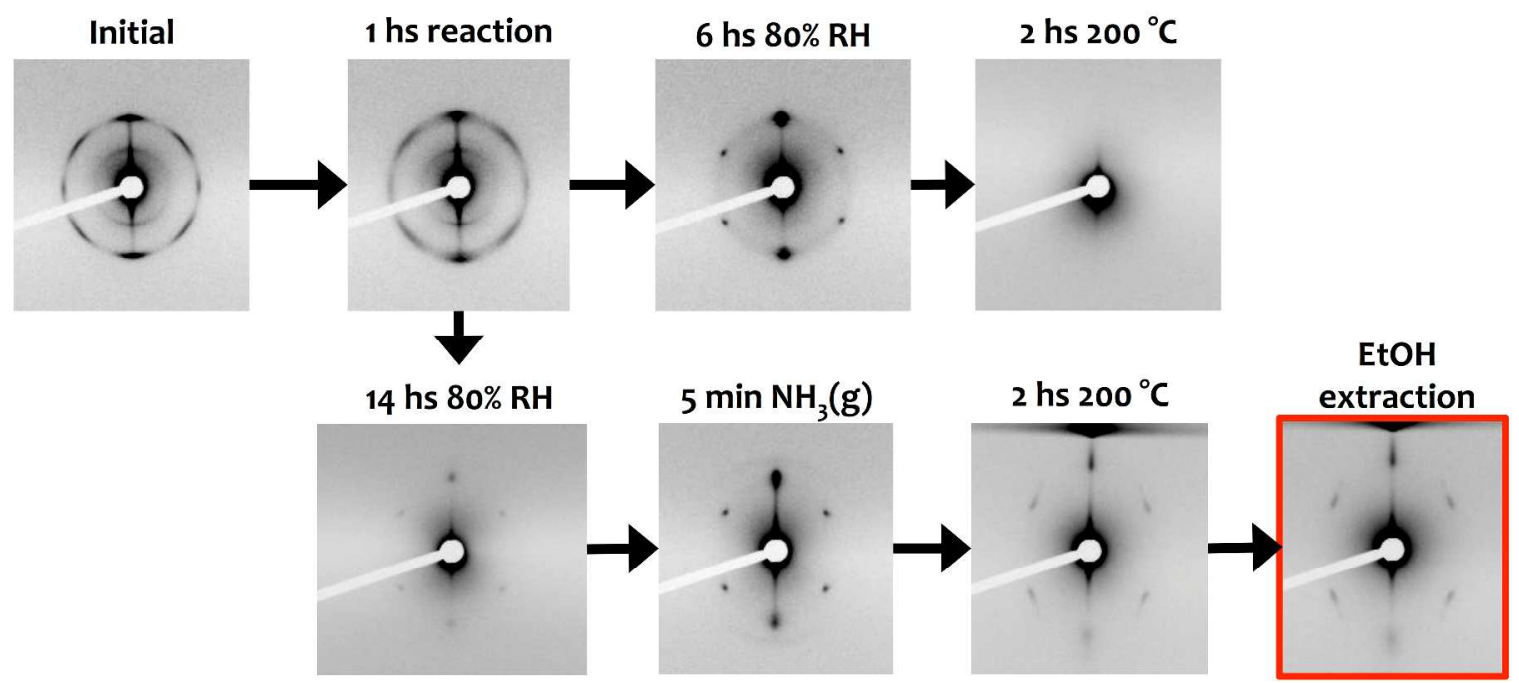

Figure 3: In situ 2D-SAXS characterization of mesoporous thin films prepared with $\mathrm{TiCl}_{4}$ and $\mathrm{Brij}$ 56 under different conditions, as indicated in the diagram. The complete set of conditions studied can be found in Figure S3 (SI).

The in situ experiments presented above reinforce the idea that the $p 6 \mathrm{~mm}$ structure is very unstable for $\mathrm{TiO}_{2}$, difficult to stabilize and easily lost after thermal treatments. Taking into account that the $p 6 \mathrm{~mm}$ structure is routinely obtained for $\mathrm{SiO}_{2}$, a possible explanation for such behavior could be related to the combination of the mechanical properties of the $p 6 \mathrm{~mm}$ phase ${ }^{42}$ with the higher reactivity of $\mathrm{Ti}$ and the tendency of $\mathrm{TiO}_{2}$ to crystallize at relatively low temperatures. ${ }^{10,12}$ In addition, topological effects might affect the $\mathrm{TiO}_{2}$ densification process that takes place upon higher temperature treatment. Densification and eventual crystallization has been shown to proceed through diffusive sintering in MTTF. ${ }^{43}$ For $\operatorname{Im} 3 m$ cubic phase, the most usual for MTTF, the structure's open porosity is known for restricting densification/crystallite growth and thus can minimize the mesostructure collapse at moderate temperatures. ${ }^{12}$ In contrast, the topology of the $p 6 \mathrm{~mm}$ structure allows for a faster densification and eventual growth of larger anisotropic anatase crystals. Finally, the possible effect of the different synthesis and post synthesis conditions onto the nucleation and growth of anatase nanocrystals in the oxide's walls, as has been demonstrated previously, ${ }^{12,44}$ cannot be ruled out. In fact, differences in the crystals' formation processes could lead to preferential disruption of certain mesophases. These working hypotheses are currently under study in our group and will be expanded in the future.

The results discussed in this section emphasize the importance of the in situ characterization for materials' design. In particular, it is shown that measurements performed 
after every step of the material's processing help to understand the key points of the treatment and to design new procedures in order to obtain the desired material properties, allowing a more rational approach to develop reproducible and well-understood synthesis protocols.

\section{Cross-characterization of the $p 6 \mathrm{~mm} \mathrm{TiO}_{2}$ sample}

For the case in which the $p 6 \mathrm{~mm}$ structure was obtained $\left(\mathrm{TiCl}_{4}-\mathrm{Brij56}\right.$ system after calcination at mild temperature followed by ethanol extraction, Figure 3 last panel), the obtained mesoporous oxide was further characterized ex situ by means of TEM and EEP. The obtained results are shown in Figure 4.
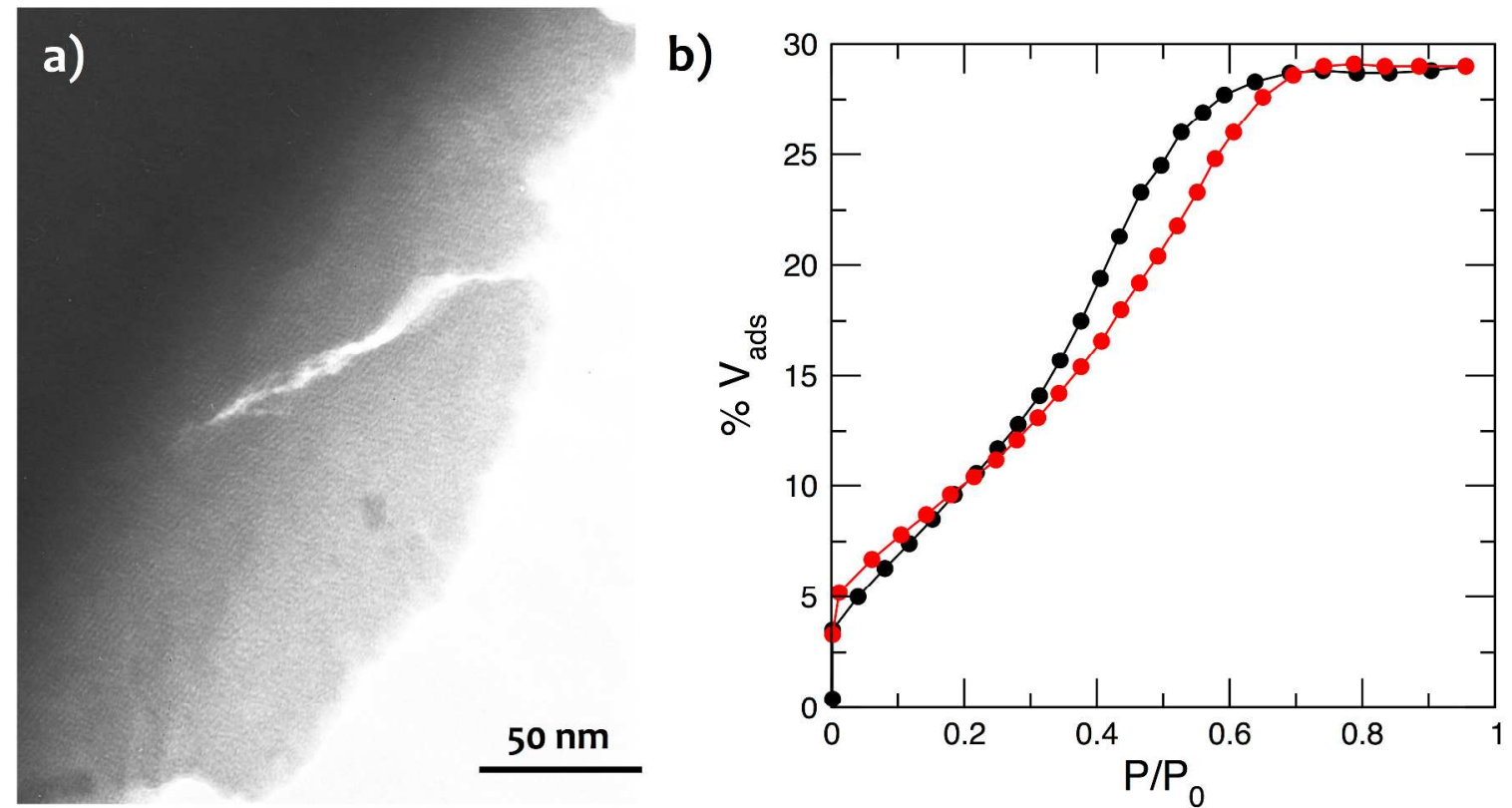

Figure 4: Ex situ characterization of the mesoporous $\mathrm{TiO}_{2}$ thin film corresponding to the in situ 2D-SAXS marked in red in Figure 3: (a) TEM image; (b) EEP isotherm showing the adsorption (red) and desorption (black) branches.

The TEM image (Figure 4a) shows areas in which the expected channels are observed; however, in other areas the order is not clear. The obtained adsorption-desorption isotherm (Figure 4b) reflects the low porosity of the sample, consistent with a 2D-hexagonal arrangement of the pores, where the accessibility is limited by geometrical restrictions. ${ }^{45}$ Further analysis of the isotherms shows that it corresponds to a type IV isotherm according to the IUPAC classification, and is characteristic of mesoporous materials. ${ }^{46}$ However, the hysteresis loop obtained is not the $\mathrm{H} 1$ type that would be expected for systems with cylindrical pores without constrictions (corresponding to a purely mesoporous $p 6 \mathrm{~mm}$ structure, as suggested by 2D-SAXS 
characterization). ${ }^{19}$ Indeed, the hysteresis loop resembles an H2-type, corresponding to a network of constricted pores with a distribution in size, connected by narrower necks. ${ }^{19}$ It can be seen that the adsorption branch is smoother that would be expected for independent cylindrical pores, since it does not present the abrupt jump in adsorption on the onset of capillary condensation. This suggests the presence of constrictions in the pores. However, such interconnectivity effects do not result in appreciable cavitation or percolation in the desorption branch. Another possibility is that a non-uniform pore diameter along the pore axis is responsible for the type $\mathrm{H} 2$ hysteresis loop obtained. A similar observation has been reported previously for $\mathrm{N}_{2}$ adsorption in titania mesoporous thin films. ${ }^{39}$ Overall, the obtained sorption behavior is consistent with cylindrical pores with a certain degree of interconnectivity or nonuniform pore size. This result is in line with the TEM image in Figure 4a, and supports that the structure of the mesoporous film is not purely $p 6 \mathrm{~mm}$.

In summary, the in situ characterization results indicate that even for a case in which a clear 2D-SAXS pattern of 2D-hexagonal pore arrangement was observed, some areas of the sample remain disordered. These results point to the intrinsic instability of the $p 6 \mathrm{~mm}$ structure and the difficulty to obtain a pure 2D-hexagonal structure. In addition, the obtained results emphasize the importance of using complementary experimental techniques to unequivocally assign structures of mesoporous materials.

\section{Conclusions}

A wide variety of MTTF using different precursors, templates, aging conditions and stabilization treatments were prepared, based on literature reported procedures, in order to obtain the $p 6 \mathrm{~mm} 2 \mathrm{D}$ porous structure. The results of cross characterization by electron microscopy and small angle X-ray diffraction demonstrated that the desired phase is hard to stabilize in the mesoporous material. In order to understand the key parameters that define the final structure, in situ 2D-SAXS experiments were designed and performed. By such experiments, a $p 6 \mathrm{~mm}$ phase was identified under several deposition conditions. When the mesophase evolution during the film synthesis and processing stages was monitored, it was demonstrated that the key parameters that affect the ordering are the post-synthesis exposure to humidity and, mainly, the thermal treatment. Thus, it was possible to stabilize the desired mesophase by a combination of high $\mathrm{RH}$ aging, exposure to $\mathrm{NH}_{3}$ vapors to consolidate the framework and a gentle thermal treatment (up to $200^{\circ} \mathrm{C}$ ). But even in this case, the obtained MTTF did not 
present a fully ordered $p 6 \mathrm{~mm}$ structure, as was observed by electron microscopy and EEP measurements. Particularly, the sensitivity to thermal treatment concluded from this work is relevant envisioning (photo)electrochemical applications of the $p 6 \mathrm{~mm}$ MTTF, since they require a controlled crystallinity of the oxide matrix only achievable by thermal treatment at high temperatures $\left(400-600^{\circ} \mathrm{C}\right)$. This work demonstrates that simple deposition techniques are not adequate to obtain $p 6 \mathrm{~mm}$ ordered structures for crystalline MTTF, suggesting that more complex post-treatment methodologies previously presented in the literature $\mathrm{e}^{16,17}$ are the ones to go for this type of material.

Finally, the potentiality of in situ 2D-SAXS measurements as a technique to design synthesis and, particularly, post synthesis procedures was demonstrated. Monitoring the mesostructure evolution after every step of the material's processing by in situ 2D-SAXS allowed determining the key parameters that affect the ordering of the MTTF. This type of experiments could be useful to design new synthetic procedures, in order to obtain specific properties of the final mesoporous material. By this means, a more rational approach to develop reproducible and well-understood synthesis protocols can be achieved.

\section{Supporting Information}

Complete set of MTTF synthesis conditions and complete set of in situ 2D-SAXS results.

\section{Acknowledgements}

This work was supported by ANPCyT (PICT 2012-0111, 2012-2087 and 2015-3526), CONICET (PIP 00044CO) and LNLS (SAXS1-14176 and 15956 projects).

Ianina Violi and Paula Steinberg are acknowledged for their experimental assistance during in situ SAXS experiments. EGS acknowledges CONICET for a doctoral fellowship.

\section{References}

1. Schneider, J.; Matsuoka, M.; Takeuchi, M.; Zhang, J.; Horiuchi, Y.; Anpo, M.; Bahnemann, D. W., Understanding TiO2 Photocatalysis: Mechanisms and Materials. Chem. Rev. 2014, 114, 9919-9986.

2. Ismail, A. A.; Bahnemann, D. W., Mesoporous Titania Photocatalysts: Preparation, Characterization and Reaction Mechanisms. J. Mater. Chem. 2011, 21, 11686-11707.

3. Wu, K. C. W.; Jiang, X.; Yamauchi, Y., New Trend on Mesoporous Films: Precise Controls of One-Dimensional (1d) Mesochannels toward Innovative Applications. J. Mater. Chem. 2011, 21, 8934-8939. 
4. Zhou, W.; Fu, H., Mesoporous TiO2: Preparation, Doping, and as a Composite for Photocatalysis. ChemCatChem 2013, 5, 885-894.

5. Pan, J. H.; Zhao, X. S.; Lee, W. I., Block Copolymer-Templated Synthesis of Highly Organized Mesoporous TiO2-Based Films and Their Photoelectrochemical Applications. Chem. Eng. J. 2011, 170, 363-380.

6. Zhang, R.; Elzatahry, A. A.; Al-Deyab, S. S.; Zhao, D., Mesoporous Titania: From Synthesis to Application. Nano Today 2012, 7, 344-366.

7. Yun, H. S.; Miyazawa, K.; Zhou, H. S.; Honma, I.; Kuwabara, M., Synthesis of Mesoporous Thin Tio2 Films with Hexagonal Pore Structures Using Triblock Copolymer Templates. Adv. Mater. 2001, 13, 1377-1380.

8. Grosso, D.; Balkenende, A. R.; Albouy, P. A.; Ayral, A.; Amenitsch, H.; Babonneau, F., Two-Dimensional Hexagonal Mesoporous Silica Thin Films Prepared from Block Copolymers: Detailed Characterization and Formation Mechanism. Chem. Mater. 2001, 13, 1848-1856.

9. Li, W.; Wu, Z.; Wang, J.; Elzatahry, A. A.; Zhao, D., A Perspective on Mesoporous TiO2 Materials. Chem. Mater. 2014, 26, 287-298.

10. Soler-Illia, G. J. A. A.; Angelomé, P. C.; Fuertes, M. C.; Grosso, D.; Boissiere, C., Critical Aspects in the Production of Periodically Ordered Mesoporous Titania Thin Films. Nanoscale 2012, 4, 2549-2566.

11. Brinker, C. J.; Lu, Y.; Sellinger, A.; Fan, H., Evaporation-Induced Self-Assembly: Nanostructures Made Easy. Adv. Mater. 1999, 11, 579-585.

12. Angelomé, P. C.; Andrini, L.; Calvo, M. E.; Requejo, F. G.; Bilmes, S. A.; Soler-Illia, G. J. A. A., Mesoporous Anatase Tio2 Films: Use of Ti K Xanes for the Quantification of the Nanocrystalline Character and Substrate Effects in the Photocatalysis Behavior. J. Phys. Chem. C 2007, 111, 10886-10893.

13. Crepaldi, E. L.; Soler-Illia, G. J. A. A.; Grosso, D.; Cagnol, F.; Ribot, F.; Sánchez, C., Controlled Formation of Highly Organized Mesoporous Titania Thin Films: From Mesostructured Hybrids to Mesoporous Nanoanatase TiO2. J. Am. Chem. Soc. 2003, 125, 9770-9786.

14. Wei, T.-C.; Hillhouse, H. W., Mass Transport and Electrode Accessibility through Periodic Self-Assembled Nanoporous Silica Thin Films. Langmuir 2007, 23, 5689-5699.

15. Carreon, M. A.; Choi, S. Y.; Mamak, M.; Chopra, N.; Ozin, G. A., Pore Architecture Affects Photocatalytic Activity of Periodic Mesoporous Nanocrystalline Anatase Thin Films. J. Mater. Chem. 2007, 17, 82-89.

16. Das, S.; Wu, Q.; Garlapalli, R. K.; Nagpure, S.; Strzalka, J.; Jiang, Z.; Rankin, S. E., In Situ Gisaxs Investigation of Pore Orientation Effects on the Thermal Transformation Mechanism in Mesoporous Titania Thin Films. J. Phys. Chem. C 2014, 118, 968-976.

17. Das, S.; Nagpure, S.; Garlapalli, R. K.; Wu, Q.; Islam, S. Z.; Strzalka, J.; Rankin, S. E., Pore Orientation Effects on the Kinetics of Mesostructure Loss in Surfactant Templated Titania Thin Films. Phys. Chem. Chem. Phys. 2016, 18, 2896-2905.

18. Ceratti, D. R.; Faustini, M.; Sinturel, C.; Vayer, M.; Dahirel, V.; Jardat, M.; Grosso, D., Critical Effect of Pore Characteristics on Capillary Infiltration in Mesoporous Films. Nanoscale 2015, 7, 5371-5382.

19. Lowell, S.; Shields, J. E.; Thomas, M. A.; Thommes, M., Characterization of Porous Solids and Powders: Surface Area, Pore Size and Density. Springer Netherlands, 2004.

20. Thommes, M.; Cychosz, K. A., Physical Adsorption Characterization of Nanoporous Materials: Progress and Challenges. Adsorption 2014, 20, 233-250.

21. Cimino, R.; Cychosz, K. A.; Thommes, M.; Neimark, A. V., Experimental and Theoretical Studies of Scanning Adsorption-Desorption Isotherms. Colloids Surf. A 2013, 437, 76-89. 
22. Miyata, H.; Fukushima, Y.; Okamoto, K.; Takahashi, M.; Watanabe, M.; Kubo, W.; Komoto, A.; Kitamura, S.; Kanno, Y.; Kuroda, K., Remarkable Birefringence in a TiO2-SiO2 Composite Film with an Aligned Mesoporous Structure. J. Am. Chem. Soc. 2011, 133, 1353913544.

23. Miyata, H.; Fukushima, Y.; Kanno, Y.; Hayase, S.; Hara, S.; Watanabe, M.; Kitamura, S.; Takahashi, M.; Kuroda, K., Mesoporous Tio2 Films with Regularly Aligned Slit-Like Nanovoids. J. Mater. Chem. C 2015, 3, 3869-3875.

24. Zhao, D.; Yang, P.; I. Margolese, D.; D. Stucky, G., Synthesis of Continuous Mesoporous Silica Thin Films with Three-Dimensional Accessible Pore Structures. Chem. Comm. 1998, 24992500.

25. Klotz, M.; Albouy, P.-A.; Ayral, A.; Ménager, C.; Grosso, D.; Van der Lee, A.; Cabuil, V.; Babonneau, F.; Guizard, C., The True Structure of Hexagonal Mesophase-Templated Silica Films as Revealed by X-Ray Scattering: Effects of Thermal Treatments and of Nanoparticle Seeding. Chem. Mater. 2000, 12, 1721-1728.

26. Alberius, P. C. A.; Frindell, K. L.; Hayward, R. C.; Kramer, E. J.; Stucky, G. D.; Chmelka, B. F., General Predictive Syntheses of Cubic, Hexagonal, and Lamellar Silica and Titania Mesostructured Thin Films. Chem. Mater. 2002, 14, 3284-3294.

27. Choi, S. Y.; Mamak, M.; Coombs, N.; Chopra, N.; Ozin, G. A., Thermally Stable TwoDimensional Hexagonal Mesoporous Nanocrystalline Anatase, Meso-Nc-TiO2: Bulk and CrackFree Thin Film Morphologies. Adv. Funct. Mater. 2004, 14, 335-344.

28. Koganti, V. R.; Dunphy, D.; Gowrishankar, V.; McGehee, M. D.; Li, X.; Wang, J.; Rankin, S. E., Generalized Coating Route to Silica and Titania Films with Orthogonally Tilted Cylindrical Nanopore Arrays. Nano Lett. 2006, 6, 2567-2570.

29. Yang, P.; Zhao, D.; Margolese, D. I.; Chmelka, B. F., Generalized Syntheses of Large-Pore Mesoporous Metal Oxides with Semicrystalline Frameworks. Nature 1998, 396, 152-155.

30. Yang, P.; Zhao, D.; Margolese, D. I.; Chmelka, B. F.; Stucky, G. D., Block Copolymer Templating Syntheses of Mesoporous Metal Oxides with Large Ordering Lengths and Semicrystalline Framework. Chem. Mater. 1999, 11, 2813-2826.

31. Nagpure, S.; Das, S.; Garlapalli, R. K.; Strzalka, J.; Rankin, S. E., In Situ Gisaxs Investigation of Low-Temperature Aging in Oriented Surfactant-Mesostructured Titania Thin Films. J. Phys. Chem. C 2015, 119, 22970-22984.

32. Innocenzi, P.; Malfatti, L.; Kidchob, T.; Costacurta, S.; Falcaro, P.; Piccinini, M.; Marcelli, A.; Morini, P.; Sali, D.; Amenitsch, H., Time-Resolved Simultaneous Detection of Structural and Chemical Changes During Self-Assembly of Mesostructured Films. J. Phys. Chem. C 2007, 111, 5345-5350.

33. Grosso, D.; Babonneau, F.; Sanchez, C.; Soler-Illia, G. J. A. A.; Crepaldi, E. L.; Albouy, P. A.; Amenitsch, H.; Balkenende, A. R.; Brunet-Bruneau, A., A First Insight in the Mechanisms Involved in the Self-Assembly of $2 \mathrm{~d}$-Hexagonal Templated $\mathrm{SiO} 2$ and $\mathrm{TiO} 2$ Mesostructured Films During Dip-Coating. J Sol-Gel Sci. Technol. 2003, 26, 561-565.

34. Doshi, D. A.; Gibaud, A.; Goletto, V.; Lu, M.; Gerung, H.; Ocko, B.; Han, S. M.; Brinker, C. J., Peering into the Self-Assembly of Surfactant Templated Thin-Film Silica Mesophases. J. Am. Chem. Soc. 2003, 125, 11646-11655.

35. Grosso, D.; Cagnol, F.; Soler-Illia, G. J. A. A.; Crepaldi, E. L.; Amenitsch, H.; BrunetBruneau, A.; Bourgeois, A.; Sanchez, C., Fundamentals of Mesostructuring through EvaporationInduced Self-Assembly. Adv. Funct. Mater. 2004, 14, 309.

36. Grosso, D.; Soler-Illia, G. J. A. A.; Crepaldi, E. L.; Cagnol, F.; Sinturel, C.; Bourgeois, A.; Brunet-Bruneau, A.; Amenitsch, H.; Albouy, P. A.; Sanchez, C., Highly Porous TiO2 Anatase 
Optical Thin Films with Cubic Mesostructure Stabilized at $700^{\circ} \mathrm{C}$. Chem. Mater. 2003, 15, 45624570.

37. Boissiere, C.; Grosso, D.; Lepoutre, S.; Nicole, L.; Bruneau, A. B.; Sanchez, C., Porosity and Mechanical Properties of Mesoporous Thin Films Assessed by Environmental Ellipsometric Porosimetry. Langmuir 2005, 21, 12362-12371.

38. Grosso, D.; Soler-Illia, G. J. A. A.; Babonneau, F.; Sanchez, C.; Albouy, P. A.; BrunetBruneau, A.; Balkenende, A. R., Highly Organized Mesoporous Titania Thin Films Showing MonoOriented 2d Hexagonal Channels. Adv. Mater. 2001, 13, 1085-1090.

39. Wu, Q. L.; Rankin, S. E., Tuning the Wall Thickness and Pore Orientation in Mesoporous Titania Films Prepared with Low-Temperature Aging. J Sol-Gel Sci. Technol. 2011, 60, 81-90.

40. Angelomé, P. C.; Fuertes, M. C.; Soler-Illia, G. J. A. A., Multifunctional, Multilayer, Multiscale: Integrative Synthesis of Complex Macro and Mesoporous Thin Films with Spatial Separation of Porosity and Function. Adv. Mater. 2006, 18, 2397-2402.

41. Bagshaw, S. A.; Prouzet, E.; Pinnavaia, T. J., Templating of Mesoporous Molecular-Sieves by Nonionic Polyethylene Oxide Surfactants. Science 1995, 269, 1242-1244.

42. Fan, H.; Hartshorn, C.; Buchheit, T.; Tallant, D.; Assink, R.; Simpson, R.; Kissel, D. J.; Lacks, D. J.; Torquato, S.; Brinker, C. J., Modulus-Density Scaling Behaviour and Framework Architecture of Nanoporous Self-Assembled Silicas. Nature Mater. 2007, 6, 418-423.

43. Violi, I. L.; Perez, M. D.; Fuertes, M. C.; Soler-Illia, G. J. A. A., Highly Ordered, Accessible and Nanocrystalline Mesoporous Tio2 Thin Films on Transparent Conductive Substrates. ACS Appl. Mater. Interfaces 2012, 4, 4320-4330.

44. Innocenzi, P.; Malfatti, L.; Kidchob, T.; Enzo, S.; Ventura, G. D.; Schade, U.; Marcelli, A., Correlative Analysis of the Crystallization of Sol-Gel Dense and Mesoporous Anatase Titania Films. J. Phys. Chem. C 2010, 114, 22385-22391.

45. Richman, E. K.; Brezesinski, T.; Tolbert, S. H., Vertically Oriented Hexagonal Mesoporous Films Formed through Nanometre-Scale Epitaxy. Nature Mater. 2008, 7, 712-717.

46. Sing, K. S. W., Reporting Physisorption Data for Gas/Solid Systems with Special Reference to the Determination of Surface Area and Porosity. Pure Appl. Chem. 1985, 57, 603619. 


\section{TOC Graphic}

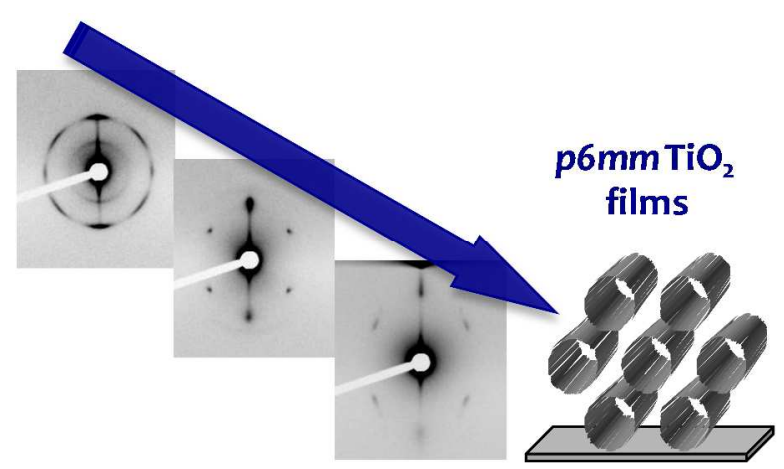

